\title{
Multifaceted strategy needed to improve dentists' adherence to evidence-based guidelines
}

\author{
Abstracted from \\ Mettes TG, van der Sanden WJ, Bronkhorst E, Grol RP, Wensing M, Plasschaert AJ. \\ Impact of guideline implementation on patient care: a cluster RCT. J Dent Res 2010; 89: 71-76 \\ Address for correspondence: Department of Preventive and Restorative Dentistry, \\ College of Oral Sciences, Radboud University, Nijmegen Medical Centre, PO Box 9101, 6500 HB Nijmegen, \\ The Netherlands. E-mail: d.mettes@dent.umcn.nl
}

\section{Question: In dental practice are multifaceted guideline-implementation strategies more effective than dissemination alone?}

Design This was a cluster-randomised clinical trial (RCT) of incomplete block design.

Intervention The interventions comprised an online 'patient-simulated clinical case' assessment, guideline dissemination, an interactive educational meeting, and flow chart reminders. All participants received feedback on individual as well as group scores for the patient-simulated clinical case assessment. Reminders with particular information and guideline-algorithm flow diagrams were provided 2 months before post-intervention measurements.

Outcome measure The primary outcome measure was guidelineadherent recall interval assignment, and the secondary outcome measure was guideline-adherent bitewing frequency prescription. Results For low-risk patients, guideline-adherent recall increased in the intervention group $(+8 \%)$, which differed from the control group (-6.1\%; P 0.01). Guideline-adherent bitewings showed mixed results.

Conclusions Multifaceted intervention had a moderate but relevant effect on the performance of general dental practitioners, which is consistent with other findings in primary care.

\section{Commentary}

Evidence-based clinical practice guidelines have the potential to improve the care received by patients by promoting interventions of proven benefit and discouraging ineffective interventions. Findings from health services research suggest, however, that there is a failure to routinely translate research findings into daily practice, which leads to a gap between the best available evidence and routine clinical practice. Studies have shown that traditional dissemination techniques such as peer-reviewed publications or continuing education do not lead to a change in practice by healthcare providers. ${ }^{1}$ This is not because healthcare professionals are not trying to do the best for their patients - a wide range of factors can influence how a professional makes decisions, including an individual's motivational predisposition to change, economics, local politics and organisational barriers. Thus, interventions designed to change professional behaviour or improve quality should have a sound theoretical basis, as did the clinical trial by Mettes and colleagues. These authors conducted a cluster RCT to measure the impact of a multifaceted strategy of change-management, to ensure individual tailoring of oral and radiographic bitewing examination frequency.

With a decline in the overall prevalence of oral diseases in the Western world, we should no longer be routinely using the same frequency for oral and radiographic exams for all patients. By painting all patients with the same brush, those at low risk of caries and periodontal disease are likely to be over-treated, whereas those at high risk may be under-treated.

The interventions were a combination of passive knowledge transfer (guideline dissemination, reminders) and active exchange (online clinical case assessment, interactive educational meetings and individualised feedback). Unfortunately, the change in behaviour was minimal. There was no significant reduction in the frequency of bitewings for low-risk patients; there was, however, a small increase in the length of time between oral examinations for this group. This could be a result of, in part, the choice of 'control' intervention. This group received the same risk-management strategies, using management of asymptomatic third molars rather than frequency of oral examinations. (The outcome was the same for both groups - that being adherence to recall and bitewing frequency guidelines). The use of risk management concepts may have made this group more aware of risk management on the whole, accounting for some confounding of the results. The other potential confounder is that most dentists were already compliant with guidelines for high-risk patients.

Mettes' groups should be congratulated on tackling the issue of changing practitioner behaviour. Although much work remains to be done, they have shown that, despite the difficulties, it is not impossible to teach an old dog new tricks.

\section{Debora C Matthews}

Department of Dental Clinical Sciences, Dalhousie University, Halifax, Nova Scotia, Canada

1. Grimshaw J, Eccles M, Tetroe J. Implementing clinical guidelines: current evid ence and future im plications. J Cont Ed in Health Professions 2004; 24 (suppl. 1): S31-S37 